\title{
ENTREVISTA CON ENRIOUE GARCÍA: IMPASES EN EL DESARROLLO DE AMÉRICA LATINA
}

Entrevista realizada por el Prof. Dr. Pedro Dallari, del Instituto de Relaciones Internacionales y coordinador del Centro Iberoamericano (Ciba) y de la Cátedra José Bonifácio de la Universidad de São Paulo (USP) al economista boliviano Enrique García, en diciembre de 2019. La entrevista forma parte de las actividades realizadas en el ámbito de la Cátedra José Bonifácio.

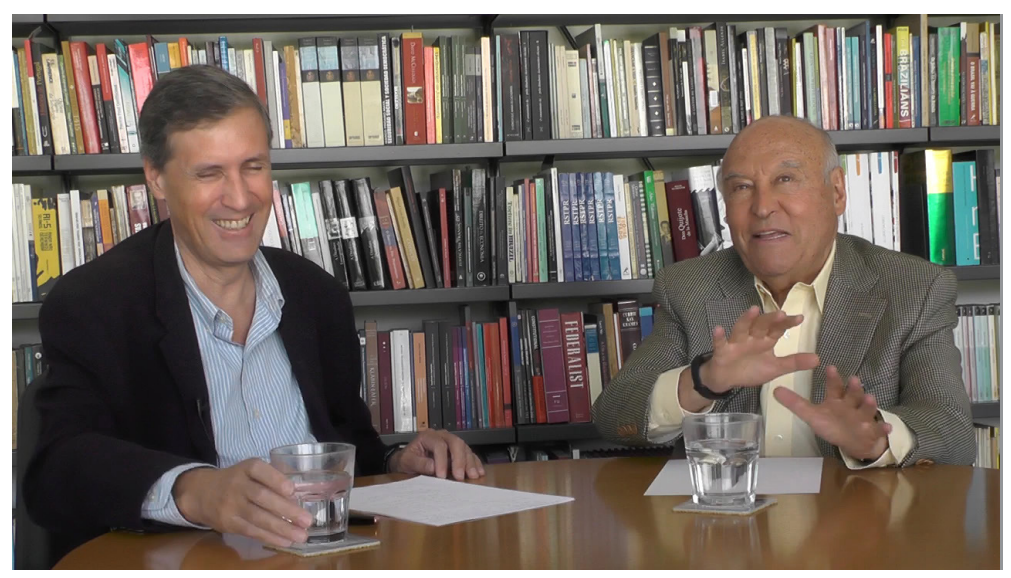

Foto: Prof. Dr. Pedro B. A. Dallari (izq.) y Dr. Enrique García (der.). Fuente: Archivo CIBA-USP).

Prdeo B. A. Dallari

Profesor titular del Instituto de Relaciones Internacionales. Coordinador del Centro Iberoamericano (Ciba) y de la Cátedra José Bonifácio de la Universidade de São Paulo (USP, Brasil). 
La realización y publicación de esta entrevista con el economista boliviano Luis Enrique García Rodríguez, personalidad destacada en el marco de las relaciones internacionales y latinoamericanas, es fruto de la fructífera colaboración que mantienen el Centro de Estudios Brasileños de la Universidad de Salamanca (CEB-USAL) y el Centro Iberoamericano de la Universidad de São Paulo (CIBA-USP). En ese contexto de cooperación, la Revista de Estudios Brasileños (REB), fundada por el CEB y que tiene la colaboración de la USP, ha publicado en los últimos años las entrevistas que he realizado anualmente con los titulares de la Cátedra José Bonifácio de la USP, programa de apoyo a la investigación gestionado por el CIBA. Es el caso de las entrevistas de Beatriz Paredes, importante figura de la vida política y diplomática mexicana, que estuvo al frente de la Cátedra José Bonifácio en 2017¹ y de la expresidenta de Costa Rica, Laura Chinchilla, catedrática en $2018^{2}$. En esta ocasión, es el momento de conocer las opiniones de L. Enrique García R., el catedrático que asumió el cargo en 2019.

Aunque no son brasileños, las entrevistas tienen en común la finalidad de presentar la visión de los catedráticos sobre la realidad de América Latina y, en ese escenario, de Brasil, teniendo en cuenta, precisamente, la experiencia resultante de su estancia en São Paulo, conviviendo con la comunidad de profesores, investigadores y alumnos de una de las más prestigiosas universidades del país. La perspectiva de los catedráticos, que, por un lado, es extranjera, pero que, por otro, es el resultado de la inmersión en el ambiente académico brasileño, se ha revelado una fuente preciosa de conocimiento sobre Brasil, en gran medida debido a la peculiaridad de que los entrevistados son personas cuya relevancia en el espacio público demuestra la capacidad de entender la sociedad en la que viven.

Luis Enrique García Rodríguez es la séptima personalidad, la primera de nacionalidad boliviana, en ocupar la Cátedra José Bonifácio. Antes de él y de Laura Chinchilla y Beatriz Paredes, fueron titulares de la Cátedra el expresidente chileno Ricardo Lagos (2013), el economista uruguayo Enrique Iglesias (2014), que dirigió el Banco Interamericano de Desarrollo (BID) y la Secretaría General Iberoamericana (Segib), la escritora brasileña Nélida Piñón (2015), expresidenta de la Academia Brasileña de Letras (ABL), y el expresidente del gobierno de España Felipe González (2016). Al hacer posible la presencia de esas personalidades en la USP, la Cátedra tiene el objetivo de ofrecer a los investigadores la oportunidad de beneficiarse, de forma más intensa y prolongada, de la convivencia con destacados líderes políticos, sociales y culturales, permitiendo incorporar al conocimiento científico la experiencia transmitida por los catedráticos.

La producción de la Cátedra José Bonifácio se expresa principalmente a través de los libros coordinados por los catedráticos, que se publican anualmente por la editorial de la Universidad de São Paulo (Edusp). Los libros reúnen artículos en español y portugués de académicos e investigadores que trabajaron con los catedráticos, formando un importante repositorio del pensamiento sobre asuntos iberoamericanos. Todos los libros de la colección están disponibles en versión digital y con acceso gratuito al público en el sitio web de Edusp:

[http://www.livrosabertos.edusp.usp.br/edusp/catalog/category/relacoes-internacionais].

De acuerdo con las normas de la Cátedra, cada catedrático elige el tema de investigación de su periodo de titularidad, que es también el objeto del libro publicado bajo su coordinación. Respaldado por una sólida formación académica y una excepcional trayectoria en la dirección de asuntos económicos y financieros - entre muchas actividades, fue ministro de Planificación de Bolivia y presidió la CAF, el Banco de Desarrollo de América Latina -, L. Enrique García R. ha optado por centrarse en los desafíos planteados al desarrollo de América Latina en el nuevo contexto internacional. Los diversos aspectos de ese tema fueron, así, tratados por su
PALABRAS CLAVE Enrique García; Pedro Dallari; Cátedra José Bonifácio; Brasil.

PALAVRAS-CHAVE Enrique García; Pedro Dallari; Cátedra José Bonifácio; Brasil.

KEYWORDS Enrique García;

Pedro Dallari; José Bonifácio Chair; Brazil. 
grupo de investigadores, alumnos de diferentes programas de posgraduación de la USP, y en las diversas conferencias impartidas por el catedrático, así como en las otras actividades realizadas en São Paulo a lo largo de 2019.

En la tarde del viernes 29 de noviembre, en medio de las tareas de coordinación del libro que preparaba para la Cátedra, Enrique García se reunió conmigo en su despacho del CIBA. Con el testimonio de la extensa colección de obras de historia y cultura latinoamericana de la Biblioteca Ayacucho, disponible en las estanterías de la sala, conversamos sobre Brasil, América Latina y los impases para el desarrollo de la región. También sobre las actividades realizadas por el catedrático en la USP. Tras esta presentación, está la transcripción de la entrevista, que fue revisada por el propio entrevistado ${ }^{3}$. De manera simple y clara, L. Enrique García R., con su vasta experiencia de ciudadano del mundo, ofrece una valiosa ayuda para comprender una coyuntura marcada por su extrema complejidad.

Pedro B. A. Dallari: Luis Enrique García Rodríguez, boliviano, economista de formación, una leyenda del sistema financiero internacional, como me gusta decir, y una personalidad muy activa en la gobernanza internacional.

Enrique García está con nosotros en la Universidad de São Paulo (USP) como catedrático de la Cátedra José Bonifácio. La Cátedra José Bonifácio es un programa de apoyo a la investigación sobre temas de Iberoamérica y América Latina. Además, cada año tenemos en la Cátedra una gran personalidad del espacio iberoamericano. Con nosotros ya estuvo Ricardo Lagos, expresidente de Chile; Enrique Iglesias, exsecretario de la Secretaría General Iberoamericana (Segib), y expresidente del Banco Interamericano de Desarrollo (BID); Nélida Piñon, que fue la primera mujer en presidir la Academia Brasileña de Letras (ABL); después, Felipe González, que fue presidente del gobierno de España; Beatriz Paredes, una líder política, social, mexicana, extraordinaria; Laura Chinchilla, que fue presidenta de Costa Rica, y, ahora, Enrique García o don Enrique, como nos gusta llamarle aquí en la USP. Don Enrique se ha quedado con nosotros desde 2019 hasta el inicio de 2020, para dirigir un grupo de investigación e impartir conferencias, participando muy intensamente de la vida académica en general.

Me gustaría empezar preguntando lo más obvio sobre la actualidad, sobre este momento turbulento en América del Sur. ¿Qué está pasando en América del Sur? Ecuador, Chile, Bolivia, con un escenario de manifestaciones populares muy intensas. Argentina y Uruguay con cambios de partidos en la jefatura del gobierno. En Venezuela, sigue la dictadura. Hace unos años, América Latina era un continente de paz y prosperidad, pero ahora vuelve a un escenario ya vivido en el pasado. La pregunta que te hago, que también se la hice a otros líderes, es esta: ¿esta es una crisis de la democracia? ¿Está la democracia en riesgo en América Latina? ¿O es una crisis en la democracia porque la sociedad quiere más participación, más cambios, mejoras en la gobernanza en la región?

Enrique García: Muchísimas gracias, Pedro. Es un grato honor estar en esta entrevista y, particularmente, haber asumido la Cátedra durante el periodo 2019-2020. Es una experiencia espectacular: esta es una gran universidad y, además, por tener contacto, no solo académico, sino humano, con profesores y estudiantes, en este momento muy especial para América Latina; es estimulante. Tu pregunta es muy pertinente. No hay ninguna duda de que este es un momento difícil. Un momento de transición. ¿Es una crisis de la democracia o es una crisis en la democracia? Yo me arriesgaría a decir que no es una crisis de la democracia, yo la veo más como una crisis en la democracia. ¿Por qué? Porque muchos de los fenómenos que han pasado, especialmente en los últimos seis meses, en las crisis que hemos visto en Ecuador, Perú, Chile, Colombia y en mi país, Bolivia, son, en realidad, expresiones, de una u otra manera, de sociedades que demandan mayor participación, equidad social y transparencia; y que no están satisfechas con muchos de los estilos que la clase política tradicional estaba acostumbrada a practicar. También tiene mucho que ver con un cambio de época. La tecnología, que nos ha traído tantas novedades en comunicación e interacción social, hace que los interlocutores tradicionales como los parlamentos, partidos políticos y organizaciones gremiales no sean tan importantes como lo eran antes. Hoy en día, la comunicación a través de los teléfonos móviles, instrumento que nos tiene a todos tan obsesionados, hace que se pueda convocar y movilizar a la 
sociedad sin necesidad de intermediarios. Muchos de los fenómenos que estamos viviendo en las últimas semanas no han sido generados por los mecanismos tradicionales. Veamos lo sucedido en Ecuador, lo que está pasando en Chile o lo acontecido en mi país, Bolivia. Una pregunta clave: ¿quién ha sido el que ha originado las movilizaciones sociales? La respuesta es clara: la sociedad civil en la calle, integrada por estudiantes, trabajadores, amas de casa y la sociedad civil en su conjunto ¿Protestando contra qué cosas? Contra la falta de consolidación de la institucionalidad democrática. Lo negativo es que ha habido violencia. Lo positivo es que se ha dado una clara señal de que estamos en un momento de inflexión. Lo veo en ese sentido. El hecho de que varios países continúan convulsionados indica que es un momento de reflexión, en el cual debemos replantear nuestros enfoques y prioridades y determinar dónde hemos fallado. Lo que está claro es que la sociedad no acepta ni tolera la desigualdad, ya no acepta la corrupción ni la impunidad; quiere soluciones y pronto. También es evidente que la sociedad demanda mayor igualdad y equidad. El gran desafío es cómo lograrlo en un momento en el que, lamentablemente, los vientos de cola que tenía el barco de vela en que navegaba América Latina, a principios y mediados de este siglo, han cambiado de rumbo y ahora tiene que navegar con vientos de proa. ¿Entonces cómo compatibilizamos estas ansiedades con la realidad de que la economía de nuestros países, de esta América Latina, no tendrá, por unos años, el ritmo de crecimiento y de avance que tenía antes?

PD: Aprovecho para preguntarte justamente sobre esto. Tienes una experiencia extraordinaria en temas económicos. Durante casi tres décadas, tuviste una experiencia enorme en el Banco Interamericano de Desarrollo y en muchas organizaciones internacionales. Casi treinta años como presidente de CAF, el Banco de Desarrollo de América Latina. Quería que comentaras sobre la situación económica: ¿es posible retomar el desarrollo en América Latina? Y lo pregunto basándome en el proyecto, en el tema de investigación que has elegido para la Cátedra, el tema del desarrollo, pero desde una perspectiva integral, holística, como te gusta decir. Me gustaría que nos explicaras cuál es tu perspectiva. Como sabemos, cada catedrático, cuando asume la Cátedra José Bonifácio, selecciona un tema, que será el tema de la investigación.

Enrique García: Desde mi juventud, estoy profundamente comprometido con los temas vinculados con el desarrollo de América Latina. Primero, con la necesidad de que los países tengan una visión de largo plazo. Segundo, con la importancia de no ver los aspectos económicos, sociales y ambientales de forma aislada sin interrelacionarlos integralmente. Al respecto, en mi experiencia internacional de varias décadas, he podido observar que tanto los ministros del área macroeconómica y financiera como los presidentes de los bancos centrales - que están preocupados, de la situación fiscal y monetaria, de que la inflación sea baja y de que la deuda externa sea sostenible - no vinculan necesariamente estos aspectos con otros elementos fundamentales en una estrategia de desarrollo. ¿De qué manera las políticas y acciones en el ámbito macroeconómico tienen impacto en el crecimiento económico? ¿De qué tipo de crecimiento se está hablando? ¿Es un crecimiento de calidad? ¿Es un crecimiento estable, eficiente, incluyente, equitativo y sostenible? Tampoco se vinculan e interrelacionan sistemáticamente los aspectos económicos y sociales con un tema central y crítico en el mundo actual, como es la sostenibilidad ambiental. Todos estos temas fundamentales son generalmente tratados en forma aislada y no con un enfoque holístico integral, como corresponde. En mi capacidad de presidente de CAF, y ejecutivo del BID, he tenido la oportunidad de participar regularmente en reuniones de ministros y autoridades macroeconómicas de la región. En dichas reuniones, había euforia y triunfalismo en los años de bonanza; todos estaban felices, pero no se hablaba mayormente de otros temas, más allá de la macroeconomía. Lo mismo sucedía en las reuniones de autoridades del campo social o ambiental, que también se concentraban en discutir los temas sectoriales de su competencia, en forma aislada. A la luz de todo lo anterior, estoy convencido de que se necesita tener una visión y un enfoque integral para promover el desarrollo. No basta reunirse entre todos los que piensan igual y tienen profesiones e interés similares. Más bien, hay que estimular y organizar encuentros que incluyan una mezcla interdisciplinaria entre macroeconomistas, desarrollistas, actores del área social y ambiental, así como representantes de los sectores público y privado, así como de la sociedad civil. Todo ello con el propósito de lograr consensos básicos para concebir un proyecto de desarrollo con visión integral de largo plazo. Lamentablemente, por lo general, esto no sucede en América Latina. La verdad es que seguimos muy enfrascados en un modelo de ventajas comparativas tradicionales. Dios bendito que nos ha dado abundantes recursos naturales y materias primas. Sin embargo, hemos sido complacientes, ya que 
nos hemos dedicado principalmente a su explotación y exportación sin mayor diversificación y generación de valor agregado. En ese contexto, la historia se repite: cuando los vientos son favorables, los precios internacionales son altos, y el mundo crece, nos sentimos felices y nos volvemos complacientes. En efecto, no aprovechamos el momento para hacer los cambios que permitan un salto a un modelo más estable de ventajas competitivas, a través de tecnología, innovación e institucionalidad adecuada y acorde con los nuevos tiempos.

Para ser exitosos en el futuro, es fundamental un cambio de actitud para no quedar anclados en el pasado y mirar más bien el futuro, teniendo en cuenta las realidades implícitas en la cuarta revolución industrial. ¿Cuáles son las implicaciones que tiene la digitalización? ¿Qué sistemas educativos y de formación de recursos humanos van a requerirse en el futuro? ¿Qué políticas públicas deben adoptarse para dar oportunidades y medios para que la mayoría de los ciudadanos tenga posibilidades ciertas de progreso y puedan dar a sus hijos una mejor calidad de vida?

PD: ¿Y cuál es tu perspectiva? ¿Esto es posible? ¿Es viable?

Enrique García: Yo diría que es lo deseable. Pero, quiero ser franco y honesto, no lo veo fácil. Los gobiernos y principales actores de la sociedad no están pensando de esta manera. Están actuando con un enfoque parcial de corto plazo, muy vinculado a los ciclos electorales, y tratando de responder la pregunta de cómo se pueden lograr resultados rápidos en cuatro o cinco años. La realidad es que los cambios estructurales relevantes requieren más de diez años para verificar sus resultados. La actitud anterior muestra la debilidad de la institucionalidad democrática de la región al no tener mecanismos idóneos para lograr consensos sobre temas centrales que requieren una visión de desarrollo de largo plazo. Evidentemente, no todos piensan de igual manera. ¡Lo sé! Usaré términos que no me gustan, pero quizás faciliten la comprensión del problema: una cosa es un gobierno de centroderecha o derecha y otra es un gobierno de centroizquierda o izquierda; obviamente tienen diferencias ideológicas y conceptuales, pero hay ciertos aspectos y conceptos comunes, de interés nacional, que trascienden lo sectario y sobre los cuales deben lograrse consensos y acuerdos. Como manifesté al iniciar esta entrevista, en este momento, lo que se observa, y sobre lo cual hay que reflexionar y buscar soluciones, no es sobre la crisis de la democracia, sino sobre la crisis en la democracia y su institucionalidad.

PD: ¿Y cómo ve a Brasil en este contexto? Porque Brasil es la más grande economía, el país de mayor territorio, de mayor población; pero muchas veces, en mis viajes por América Latina, yo siento que hay muchas expectativas en relación con Brasil, pero que, en general, hay un poco de frustración, porque Brasil no ocupa una posición de liderazgo.

Enrique García: Pedro, tú lo has expresado muy claramente. ¿Brasil es un país? No, Brasil es un continente. Brasil tiene una importancia y trascendencia que van más allá de su dimensión territorial, población, mezcla de culturas y fortaleza económica; las características de este país hacen que tenga una responsabilidad muy grande. En efecto, gran parte de las soluciones de carácter regional requieren que Brasil sea un actor dinámico en la articulación de procesos efectivos para avanzar en la integración regional. Hubo momentos muy buenos en la historia, en los que Brasil asumió sus responsabilidades. No se puede hablar de todos los gobiernos, pero yo he trabajado muy de cerca con los gobiernos de los presidentes Cardoso y Lula. El presidente Cardoso tuvo un rol importantísimo en promover la integración regional, tanto de América del Sur como de América Latina en un sentido más amplio. A su vez, el presidente Lula trabajó con la visión de fortalecer el rol de América Latina a nivel global. Ambos presidentes fueron grandes impulsores de CAF, el Banco de Desarrollo de América Latina, durante mi presidencia de la Institución por veinticinco años. Espero con ilusión que Brasil vuelva a tener ese liderazgo y compromiso con la región. Uno de mis grandes sueños es que la integración regional se consolide y se haga realidad. La integración regional no debe verse como una cuestión ideal, sino como una manera efectiva de poder insertar a la región en el mundo, teniendo presentes las realidades del siglo XXI. Hay que estar conscientes que las cadenas productivas y de comercio, así como la relevancia geopolítica de América Latina a escala global, se hacen muy difíciles sin una integración regional pragmática y efectiva. Hoy en día, veo con mucha preocupación las crecientes 
discrepancias entre los países de la región. Como ilustración, la cercanía que se estaba logrando, después de mucho tiempo, entre México y Brasil, hoy, la veo muy difícil. Otro tema que me preocupa, ya en el ámbito suramericano, es el reciente distanciamiento entre Brasil y Argentina, que tiene serias implicaciones sobre el futuro del Mercosur. Al mismo tiempo, el resto de los esquemas de integración muestra fragmentación y debilidad institucional y operativa. Por ejemplo, se creó la Alianza del Pacífico cuando todos los gobiernos de los países fundadores tenían la misma orientación ideológica. Hoy la situación ha cambiado.

PD: Para concluir, quería que hablaras de tu presencia en Brasil, en la Cátedra. Después de tantos años como un globetrotter de la economía internacional, ahora te dedicas a la tarea académica, que es muy metódica. Estás organizando un libro, como hacen todos los catedráticos, para la colección, con aportaciones de los investigadores, de los colaboradores invitados, sobre el tema del desarrollo. ¿Qué te parece esta experiencia en la universidad?

Enrique García: Pedro, para mí, este es un momento muy importante. Desde mi juventud, me ha encantado la vida universitaria, la cátedra. En mi país, era catedrático en dos universidades, la Universidad Mayor de San Andrés y la Universidad Católica; siempre he estado muy involucrado. Obviamente, con mis funciones como presidente de CAF, no podía tener una obligación de este tipo, pero sí he estado muy vinculado con el mundo académico. Como presidente de CAF, me dediqué a establecer vínculos muy importantes con universidades a nivel global, con London School of Economics, con SciencesPo, con la Universidad de Salamanca, con universidades en todo el mundo. Desde luego, la oportunidad de estar en esta gran universidad, que, a propósito, es la número uno de América Latina, y poder tener la oportunidad de interactuar y reflexionar con gente inteligente, gente joven, sobre los problemas del mundo y de la región, es un privilegio. Han cambiado los tiempos. Creo que, en esta nueva época, los viejos están bien para ser asesores; es tiempo de dar oportunidad a los jóvenes. Entonces, para mí, esta vivencia ha sido muy importante, tanto por haber compartido con profesores, estudiantes e investigadores, así como por haber contado con el apoyo de distinguidas personalidades a nivel internacional, que están contribuyendo con sus artículos al libro que publicará la USP como parte de la Cátedra 2019-2020.

PD: Ayer me contaste que estuviste en el Museo de Arte de São Paulo y que estás aprovechando la ciudad. Además, me dijiste que una parte de tu familia está aquí en São Paulo también.

Enrique García: La verdad es que tengo mucho cariño a Brasil, porque, en toda mi carrera, he tenido mucha vinculación con el país. Desde que estaba en el Banco Interamericano de Desarrollo, como ministro de Planeamiento y Coordinación de Bolivia y, muy especialmente, como presidente de CAF, mi contacto ha sido permanente, particularmente, desde el ingreso de Brasil como país miembro de la institución durante el primer gobierno del presidente Fernando Henrique Cardoso. Estar ahora en São Paulo, gracias a la Cátedra, cuando una de mis hijas y su familia residen desde hace un año en esta ciudad, es también motivo de gran felicidad y que me hace sentir paulista. La verdad es que yo no conocía bien São Paulo, no obstante mis continuas visitas, porque venía por periodos muy cortos vinculados con gestiones de negocios o conferencias. Ahora he podido ver lo que es São Paulo; realmente, es una ciudad de primer mundo. Un hecho que me impresiona mucho es que, no obstante que tiene avenidas con grandes edificios, urbanísticamente, después de cierto número de cuadras, no se puede construir edificios altos. Se tiene esta tradición que permite mantener los barrios residenciales con los bellos árboles y vegetación, que adornan su entorno. Pocas grandes metrópolis tienen esta característica. Que hay mucho tráfico, sí lo hay, pero que es muy ordenado es una positiva realidad.

PD: Es un placer tenerlo aquí en la Universidad de São Paulo. Este proyecto es resultado de la colaboración con la Universidad de Salamanca, que mantiene un Centro de Estudios Brasileños muy importante, dirigido por el exrector de la universidad, don Ignacio Berdugo. Además, el centro publica una revista, que es la Revista de Estudios Brasileños, que es uno de los vehículos más importantes para la diseminación del conocimiento sobre Brasil. Y, por eso, este proyecto tiene esta perspectiva de generar conocimiento, pero también de diseminarlo a través de estas colaboraciones con otras universidades, y la Universidad de Salamanca tiene un papel especial. 
Enrique García: Si hay algo que yo quería reiterar es que esta gran universidad debería ampliar más su contacto internacional. Debería verse la manera de traer más estudiantes extranjeros y ampliar los programas de intercambio. Con ese propósito, debemos aprovechar el libro que se va a publicar, para presentarlo en otros países como parte de seminarios en universidades y centros académicos de la región. Al respecto, contemplamos hacerlo en Bolivia, en colaboración con la Universidad Privada Boliviana (UPB), de la cual soy miembro de su directorio, y la Academia Boliviana de Ciencias Económicas (ABCE), de la que soy miembro de número. Muchas gracias, Pedro, ipor esta grata entrevista!

PD: Nosotros sí que agradecemos las sugerencias y el apoyo en el esfuerzo por la internacionalización de la Universidad de São Paulo. Muchísimas gracias, don Enrique. 


\section{NOTAS}

${ }^{1}$ Pedro Dallari, "Beatriz Paredes: Presencia Mexicana en Brasil”, REB: Revista de Estudios Brasileños, vol. 4, n. 8 (2017), pp. 190-200, disponible en:

https://revistas.usal.es/index.php/2386-4540/article/ view/reb201748190200.

2 Pedro Dallari, "Laura Chinchilla: Actuación Destacada en Brasil", REB: Revista de Estudios Brasileños, vol. 6, n. 12 (2019), pp. 237-243, disponible en:

https://revistas.usal.es/index.php/2386-4540/article/ view/reb2019612237243.

${ }^{3}$ El video de la entrevista está disponible en [https:// tinyurl.com/tb4zfkt].

país. O Partido Social Democrata da Alemanha (SPD) abriu um processo à época para revogar a afiliação do autor.

${ }^{16}$ Termo da língua alemã que abrange a fusão da política com o entretenimento. 